\title{
Integrated Molecular Breeding for Enhanced Genetic Improvement of Climate Smart and Insect Protected Hybrid Maize in Africa
}

\author{
Okogbenin $E^{*}$ and Oikeh S \\ African Agricultural Technology Foundation, Nairobi, Kenya \\ *Corresponding author: Okogbenin E, African Agricultural Technology Foundation, Nairobi, Kenya; Tel: +254733990151; Email: E.okogbenin@aatf-africa.org
}

Received: June 18, 2021; Accepted: June 25, 2021; Published: October 25, 2021

\begin{abstract}
Africa suffers from food deficits. Droughts along with associated insect pests have contributed to reduce crop yields, particularly maize, a major staple food crop for over 300 million people in Sub- Sahara Africa (SSA). While genetic improvements have gradually increased, albeit at low speed for genetic gains through classical breeding in Africa, mitigating the effects of climate change requires exploring innovative breeding approaches to accelerate yield response at an elevated pace. Two maize projects have explored integrated molecular breeding to improve genetic gains with new hybrid maize varieties released in Africa reaching 10 tons/ha from the abysmal low production potential of 2 tons/ha previously recorded for most open pollinated varieties (OPVs) grown by smallholder farmers. A combination of marker aided breeding and transgenesis may offer a very efficient means to rapidly transforming maize food system in Africa.
\end{abstract}

\section{Introduction}

Due to climate change, droughts have contributed to reduced crop yields [1], particularly maize, a major staple food crop for over 300 million people in SSA. The role of modern breeding in enhancing adaptation and resilience against abiotic stress and associated biotic stresses induced by climate change has been increasingly substantiated by science-based facts and evidence that supports the need for continuous investments in capacity for research and development in the developing world especially in Africa where food and nutrition is still a challenge. Until few years back, open pollinated varieties (OPV) maize were principally grown in Africa by smallholder farmers who substantially engaged in subsistence agriculture. The need to rapidly attain the UN SDGs [2] related to zero hunger, good health and wellbeing, and alleviation of poverty, means that Africa needs to transform to commercial agriculture that essentially requires increasing yield levels of maize that was for a long time just about 2 tons/ha and well below the average yields of approximately 5.5 tons/ha globally. The already dire yield levels in Africa, is unfortunately being threatened by climate change that necessitated an initiative to rapidly avail Africa, highly innovative platforms through public private partnerships (PPP) that offer the state-of-the- art modern breeding opportunities. This led to the commencement of the Water Efficient Maize for Africa (WEMA) initiative over a ten-year period that was immediately followed by TELA Maize Project currently in its 4 th year of implementation. These projects, executed in a seamless stretch of continuity and gains, have explored novel technologies and strategies to rapidly develop climate smart and insect protected maize for Africa. The partnership comprises AATF, Monsanto (Bayer Crop Science), CIMMYT, and National Agricultural Research Systems (NARS) of Kenya, Uganda, Mozambique, Tanzania, and South Africa as first tier target countries and later with Ethiopia and Nigeria as second tier countries.

The Eastern and Southern African region (ESA) has a net deficit in maize. These projects have explored perhaps the most advanced modern breeding approaches on the continent that could help revolutionize agriculture for improved food and nutrition security. Both WEMA and TELA provide yet the biggest evidence of the importance of the roles of biotechnology in improving food systems and the need to invest further in research and development capacity based on modern breeding concepts. Some key significant results, lessons learnt, experience and implications for forward breeding and development of resilient crops are appraised in this paper for transformative approach to crop genetic improvement.

\section{Enriched Pedigree-based Breeding Pipeline}

Open pollinated maize has long been grown in the SSA borne out of the need to support or supplement farm family house food needs often requiring the re-use of grains as seeds. On the long run, yields are compromised with gradual decline in productivity overtime. The need to rejig African economy to fast track development and to stem increasing poverty and for modern African agriculture has justified the need for a shift from the use of OPVs to hybrid maize. Hybrid technology revolutionized maize breeding in 1920s in the Americas and has largely accounted for the phenomenal productivity levels observed for maize worldwide [3]. Africa research systems are rapidly evolving its hybrid maize breeding.

The WEMA project, to enhance capacity for hybrid maize breeding in Africa, accessed global maize germplasm through CIMMYT and 
over 700 germplasm elite lines from Monsanto (now Bayer Crop Science) breeding programs. CIMMYT’s germplasm offered rich genetic complementation for desirable traits for improved adaptation, good producibility, drought tolerance from Drought Tolerant Maize for Africa (DTMA) populations and other highly desirable traits, which were integrated through crosses with Monsanto lines having good genetics for yield enhancement and drought tolerance offered unique opportunities to improve maize germplasm for Africa. These materials have been with African national partners who explored the new enriched germplasm in classical breeding approaches to improve the genetic background of farmer-preferred maize germplasm to enhance maize performance under optimum and moderate stress conditions (24-49\% moisture stress growing conditions) in Africa.

The enriched germplasm created good genetic base for the development of good populations for molecular breeding in attempts to strengthen forward breeding and rapid genetic gains. The improved germplasm provided strong power to explore biotechnology tools or platforms to track useful genetic variation and architecture critical to accelerated breeding. WEMA and TELA facilitated access to doubled haploids (DH) facilities of CIMMYT and Monsanto, which rapidly aided the development of thousands of improved inbred lines, and single-cross and three-way hybrids which have significantly improved productivity and genetic gain in maize in recent years [4]. Results indicate that superior DH lines outperformed top pedigree-based lines under both optimum-moisture and drought stress conditions. Yield range for DH lines under optimum-moisture conditions, for example, was between 3.5-4.7 tons/ha and were superior to pedigreebased lines with yield range of 2.2-3.4 tons/ha. Similarly, under drought stress conditions, DH lines expressed yield levels of between 1.4 and 2.1 tons/ha compared to $0.06-1.2$ tons/ha for pedigree-based lines.

\section{Molecular Breeding Architecture for Fast-tracked Forward Breeding}

Both WEMA and TELA projects used a stepwise approach that explored several molecular breeding paradigms which were arched as connected pipelines to strengthen, and drive accelerated improvement in genetic gains based on a complementary and highly synergistic system. Each segment of the breeding system adopted made a significant contribution to the whole process (Figure 1). The foundational basis for the molecular breeding architecture application adopted in the two projects, was the utilization of the enriched pedigree breeding populations used to further create biparental and multi-parental quantitative trait loci (QTL) mapping populations. These populations were used to identify useful genetic factors to dissect and better understand the complex underlying basis for drought tolerance and yield in maize. SNP markers were used for molecular studies by the project consortium to identify QTLs for the primary traits of focus (i.e., drought tolerance and productivity) which are mainly influenced by polygenic inheritance expectedly. MARS was used to identify QTLs of major and minor effects for improved performance under optimum-moisture and drought stress conditions.

The ability to explore higher number of QTLs for trait improvement under marker assisted recurrent selection (MARS) efficiently helps to provide good measure of the phenotypic variance driving complex traits with better control than would be expected for marker assisted selection (MAS) that is more amenable to traits controlled by few genes. Both major and minor QTLs were mapped. Over 180 QTLs were identified in trials for traits of adaptation and resilience mainly having phenotypic variance explained (PVE) of $1.2-13.1 \%$. For traits like Maize Lethal Necrosis (MLN) disease, whose study were incorporated at the onset of the disease's outbreak in 2012, three QTLs could explain up to 40$50 \%$ of the phenotypic variance [5]. Once QTLs are efficiently mapped, MARS as a molecular breeding (MB) approach, typically minimizes the frequency of phenotyping during breeding cycles when crosses are being made to develop best haplotype combinations and gene pyramiding to accelerate genetic gain. Genomic selection (GS), as further step was also subsequently used to advance genetic gain over MARS thus, integrating more genome coverage for additional genetic factors controlling crop performance both under optimum-moisture and drought stress conditions. Results indicate that MARS and GS increased genetic gains four times over pedigree selection in the project [6].

Hybrids developed through these conventional processes were released as DroughtTEGO ${ }^{\circ}$ in the project target countries. In further progression towards improving the genetic gains of the drought tolerant hybrids developed, the inbred parental lines of the released best performing hybrids in the target countries were selected and used for trait integration for drought tolerance through genetic engineering in Bayer Crop Science facility. MON87460 (DroughtGard ${ }^{\circledR}$, CspB gene) was used for the transgenesis. Pest build-up of stem borers (Busseola fusca, Chilo partellus, and Sesamia calamistis) on the fields are often associated with drought stress, so trait integration of MON 810 (Bt gene) for insect protection of maize was done as well and hybrids evaluated for efficacy and productivity effects. Meta analyses of field data from multiple years and several locations across countries for these trait integrations have revealed $17 \%$ yield increase for MON87460 efficacy and 43\% increase in yield for MON810 efficacy (Figure 2). Further, results indicate that MON810 partially but significantly controlled the fall armyworm (Spodoptera frugiperda) insect pest in the studies.

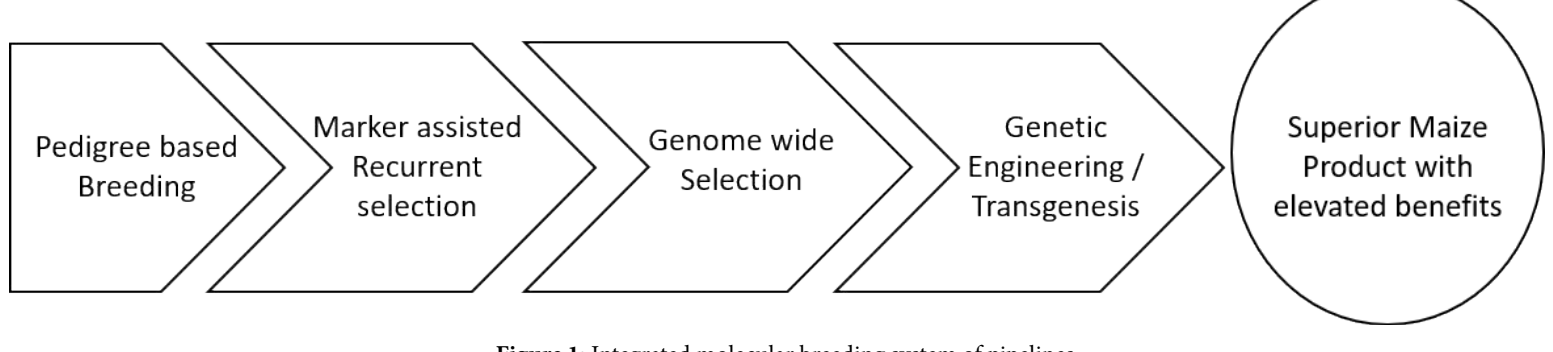

Figure 1: Integrated molecular breeding system of pipelines. 


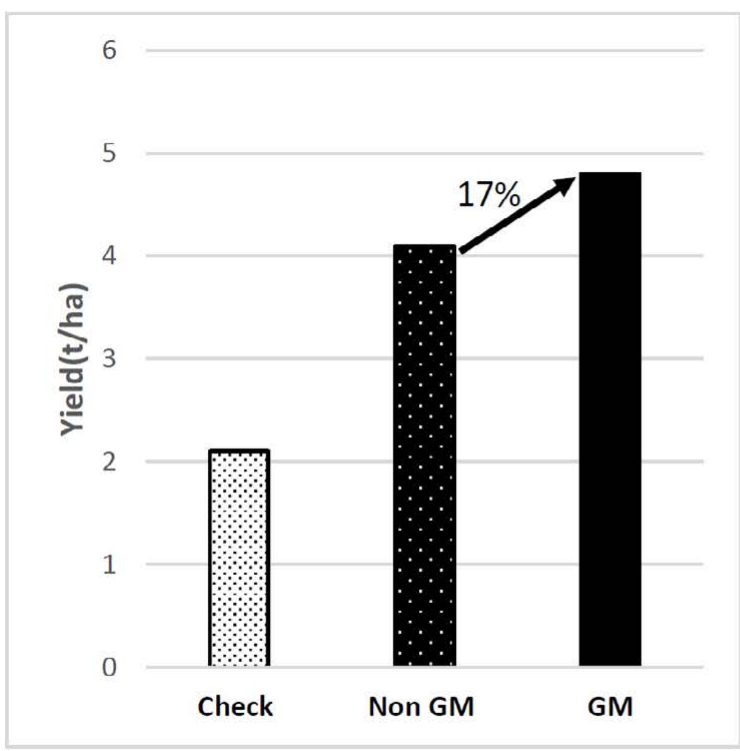

(a)
DT, MON87460 Efficacy

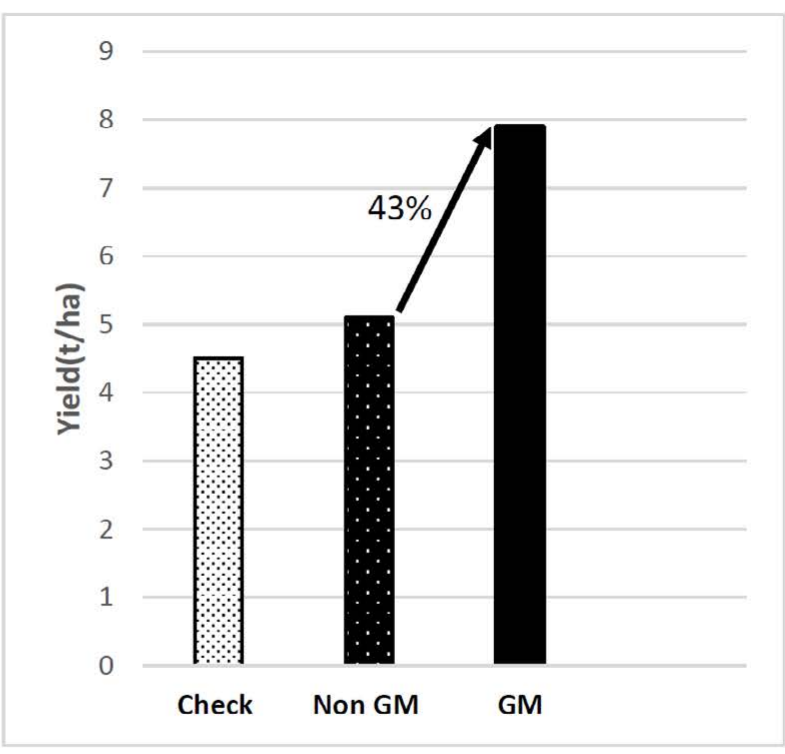

(b) Artificial Natural Stem Borer Infestation + Natural FAW

Figure 2: Efficacy of GM (Stacked MON810+MON84760) vs. Non-GM Genotypes and Commercial Checks.

Transgenic hybrid maize developed by the project has been released as TELA ${ }^{\circ}$, as its brand name. Thus far, TELA ${ }^{\circ}$ hybrids have been released in South Africa while several other promising TELA ${ }^{\circ}$ products are at advanced trial stages either for commercial release or deregulation in the other countries. Trait integration is still on-going with over 170 inbred lines traited already by the first quarter of 2021 . A total of 128 maize hybrids have been released from these projects as at 2020 (Table 1) with five being TELA ${ }^{\circ}$ within a short space of time through this PPP. Yields of the released hybrids are relatively at about 10 tons/ha under optimum-moisture conditions and nearly 5 tons/ha under drought stress conditions, exceeding current yields farmer grown varieties which were often 2 tons/ha or below when the initiatives started in 2008.

\section{Impactive Integrated Breeding as Driver to Product Scaling}

The integrated breeding strategy adopted by the projects encompassed extensive field trials to address the relevant diverse maize growing agroecologies of Africa to maximize scalability of the products in a rapid time and cost-efficient approach during scaling to other countries. WEMA wide trials were conducted first in the five first-tier target countries to assess and identify hybrids that were well suited/adapted to low-, mid- and high- altitude conditions in addition to selecting for early-, mid- and late- maturing varieties. They allowed the WEMA project to easily, based on hybrid descriptors, match DroughtTEGO ${ }^{\circledR}$ hybrids to different production zones outside the firsttier project target countries. Under the TELA project, with increased pedigree and genomic information, hybrid testing and selection of the best inbred lines and hybrids were efficiently deployed for testing in Ethiopia and Nigeria within few season trials with promising products advanced to national variety testing and then finally released within 2-3 years. In such a relatively short time, the DroughtTEGO ${ }^{\circ}$ hybrids were availed for deployment to farmers in these second-tier target countries of the project. Product scaling to other countries is expected
Table 1: Dissemination of Climate Smart Maize.

\begin{tabular}{|l|c|}
\hline Year & Climate smart maize developed and released \\
\hline 2013 & 1 \\
\hline 2014 & 22 \\
\hline 2015 & 21 \\
\hline 2016 & 55 \\
\hline 2017 & 7 \\
\hline 2018 & 11 \\
\hline 2019 & 8 \\
\hline 2020 & 3 \\
\hline Total & $\mathbf{1 2 8}$ \\
\hline
\end{tabular}

to progress through other initiatives for these products. The released DroughtTEGO ${ }^{\circ}$ hybrids in both Nigeria and Ethiopia will be further traited for insect protection genes (MON810 and MON80934) and MON87460 for drought tolerance in collaborative engagement with the regulatory authorities of both countries.

\section{Enabling Support System Needs of Africa}

Molecular breeding requires efficient infrastructural support systems and good policies to drive implementation of product development with the best strategies as articulated above. While marker aided breeding does not have inhibitory laws holding its application, this is not the case for the genetically modified (GM)based research where many countries in the Africa still have no regulatory frameworks to support its application. Where such systems are in place, cost are still prohibitory, making it more complicated to offer good entry for public-sector driven breeding initiative. A functional and easily facilitated regulatory systems are critical to building trust and confidence in the use of GM technologies and the expected products from such a process. AATF, working with its 
partnership has significantly improved the regulatory environment within Africa, getting its first GM food crop (Pod-borer Resistant [PBR] Cowpea) released for commercialization outside Republic of South Africa (RSA). It is hoped that TELA ${ }^{\circ}$ hybrids will be released soon in the other countries outside RSA.

Although MAB has a friendlier environment, the lack of capacity and limited expertise in the application of this technology could hamper the effective use of the numerous strategies it offers for rapidly developing precise and improved products for farmer and consumer needs. Increased investments in molecular breeding are, therefore, a key area requiring attention to maximize the benefits of modern breeding in Africa.

\section{Conclusion}

Biotechnology has evolved in the last few decades to address some severe limitations with classical breeding related to genetic variation, biological factors, speed, cost, and efficiency in responding to global needs of food and nutrition security as it relates to product development. The initiatives described above indicate that several molecular breeding approaches must be integrated to drive genetic gains and productivity to steer impact in huge proportions. Combining both classical breeding with molecular strategies result in better and robust products than if either strategy was used independently. The power of molecular breeding is largely dependent on the populations explored for genetic improvement as not every population is most suitable depending on the traits of focus. Given that breeding is often geared towards addressing several traits of different genetic basis (modes of inheritance and expression mechanisms), an integrated breeding approach is the most pragmatic way to addressing crop improvement needs of the 21 st century. The breeding pipelines used in these studies will hopefully, lead to an array of drought tolerant hybrid maize several years to come in efforts to mitigate climate change impacts. With genome editing strategy coming on pace, it will no doubt further enrich the integrated breeding platform now evolving to modernize African agriculture.

\section{References}

1. Meschede C (2020) The Sustainable Development Goals in Scientific Literature: A Bibliometric Overview at the Meta-Level. Sustainability 12: 4461.

2. Voss-Fels KP, Stahl A and Hickey LT (2019) Q\&A: modern crop breeding for future food security. BMC Biology 17: 18.

3. Ray RL, Fase A, Rosch E (2018) Effects of Drought on Crop Production and Cropping Areas in Texas. Agric Environ Lett 3: 170037.

4. Odiyo O, Njorogeb K, Chemining'wab G, Beyene Y (2014) Performance and adaptability of doubled haploid maize testcross hybrids under drought stress and non-stress Conditions. Int Res J Agric Sci Soil Sci 4: 150-158.

5. Semagn K, Beyene Y, Babu R, Nair S, Gowda M, et al. (2015) QTL mapping and molecular breeding for developing stress resilient maize for sub-Saharan Africa. Crop Science 55: 1449-1459.

6. Beyene Y, Semagn K, Mugo S, Tarekegne A, Babu R, et al. (2015) Genetic Gains in Grain Yield Through Genomic Selection in Eight Bi-parental Maize Populations under Drought Stress. Crop Science 55: 154-163.

\section{Citation:}

Okogbenin E, Oikeh S (2021) Integrated Molecular Breeding for Enhanced Genetic Improvement of Climate Smart and Insect Protected Hybrid Maize in Africa. Mol Genet Res Open Volume 4(2): 1-4. 